\title{
Improvement of Static Voltage Stability of 16-Bus Bali System by Optimal Placement of SVC
}

\author{
Ni Putu Agustini, I Made Wartana*, and Abraham Lomi
}

\begin{abstract}
In a power system, the reactive power imbalance is related to the stability of the static voltage because the injection of reactive power that the bus receives from the system determines the bus's capability in the system. Rapid increases in real and reactive power losses occur as the system approaches the voltage drop point or the maximum load point. It is necessary to support local and adequate reactive power to avoid system leading to be voltage collapse. This study analyzes the improvement of the margin of static voltage stability using one type of modern control equipment of shunt flexible AC transmission system (FACTS), namely the static var compensator (SVC). The controller's representations are used in the continuation power flow (CPF) process to study static voltage stability. The proposed method's effectiveness has been investigated using a practical test system, namely the Bali 16bus system, to increase the system loading capacity. The simulation was carried out by installing a modern controller in the best location, namely on bus 07 ASARI; an increase in system margin loading closed to $2 \%$ compared to the base case condition, namely $\lambda_{\max }=1,879 \mathrm{p} . \mathrm{u}$ with the voltage profile not changing significantly.
\end{abstract}

Index Terms-CPF, maximum loading point, SVC, STATCOM, voltage stability.

\section{INTRODUCTION}

Increasing demand for peak loads and power transfer between utilities has raised severe concerns about system voltages' safety in recent decades. Several major and significant faults are assumed to be responsible for the occurrence of voltage failures. Various studies have been carried out, concentrating on voltage stability to understand this phenomenon and its methods better. Several authors have proposed a voltage stability index based on several types of power flow analysis. A particular study found in the study is that the Jacobian Newton-Raphson power flow becomes single at the steady-state voltage stability limit. This stability limit is also called the critical point, often defined as when the Jacobian force flows.

Manuscript received October 18, 2020; revised January 10, 2021; accepted April 15, 2021.

Ni Putu Agustini and I Made Wartana are with Department of Electrical Engineering, National Institute of Technology, Malang, Indonesia \{e-mail: ni.putu.agustini@ lecturer.itn.ac.id; m.wartana@hotmail.com\}.

Abraham Lomi is with Renewable Energy Research Center, Department of Electrical Engineering, National Institute of Technology, Malang, Indonesia (e-mail: abraham@lecturer.itn.ac.id).

*Corresponding author.
When the reactive power imbalance impacts the voltage instability, the bus power system's durability depends on the reactive power support that the bus can receive from the system. The real and reactive power losses increase rapidly, continuing towards voltage breakdown occurring as the system approaches the point of maximum load (MLP). In this critical condition, support, local and adequate reactive power is needed. Of the two types of voltage stability based on the simulation time frame, static voltage stability involves only the solution of algebraic equations and is less extensive than dynamic voltage stability.

Most studies' ideal static voltage stability with the limits of voltage stability must be determined for various precontingency and post-contingency cases. There is a slow change in the power system in static voltage stability due to a lack of reactive power and voltage drop. This phenomenon can be accomplished by plotting the power transferred versus the receiving end's voltage, popularly called the P-V curve or "Nose" curve. As the power transfer increases, the receiving end voltage decreases, which eventually reaches a critical point (nose point). The point at which the system's reactive power is short of supply is achieved when the further increase in power transfer will cause a very rapid voltage drop. Before reaching the tipping point, a large voltage drop due to a severe loss of reactive power can be observed.

Reducing the reactive power load or increasing the reactive power is the only way to save the system from voltage collapse before reaching the voltage collapse point [2]. Considering that the voltage drop in the power system has become a crucial electrical industry problem, this study discusses the increase in static voltage by optimal placing two types of Shunt FACTS Controllers and using continuation power flow (CPF) for voltage analysis. This technique involves identifying the system equilibrium point or voltage breakdown point, where the Jacobian power flow becomes single [3],[4].

\section{LITERATURE REVIEW}

\section{A. Continuation Power Flow (CPF)}

Stress failure studies and associated tools are usually based on the following general mathematical description of the system [5]:

$$
\left.\begin{array}{l}
x=f(x, y, \lambda, p) \\
0=g(x, y, \lambda, p)
\end{array}\right\}
$$

where $X \in \mathfrak{R} n$ represents the system state variable, which relates to the generator's dynamic state, load, and other timevarying elements in the system, such as a FACTS device. At the same time, $y \in \Re n$ corresponds to algebraic variables, usually associated with transmission systems and steadystate element models, such as multiple generators and loads 
on the network. And $\lambda \in \mathfrak{R} k$ stands for an uncontrolled set of parameters that push the system to a stress collapse, which is usually used to represent system demand. The vector $p \in \mathfrak{R} k$ is used here to define system parameters controlled directly, such as shunt and series compensation levels.

Based on Eq. (1), the stress failure point can be defined, with certain assumptions, as the equilibrium point where the associated Jacobian system is singular, namely the issues $\left(x_{0}\right.$, $\left.y_{0}, \lambda_{0}, p_{0}\right) \mathrm{DxF} \mid 0$ have zero eigenvalues. This equilibrium is usually associated with the saddle-node bifurcation point as shown as the P-V curve in Fig. 1 [6].

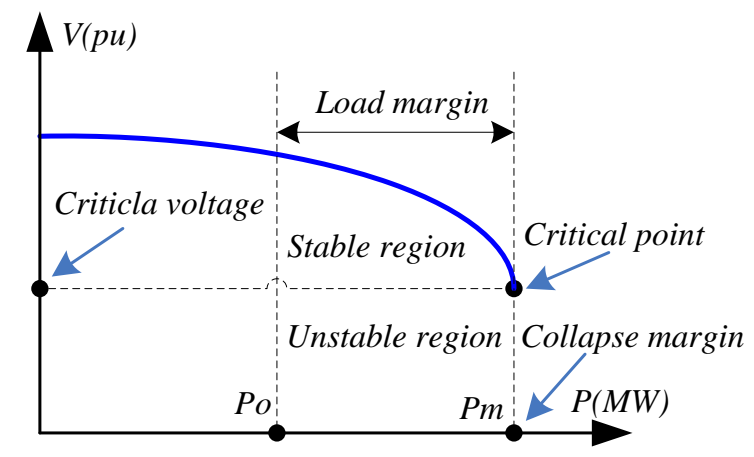

Fig. 1. P-V curve.

For a controlled set of parameters $P_{0}$, stress collapse studies typically concentrate on determining the breakdown point or bifurcation point $\left(x_{0}, y_{0}, \lambda_{0}\right)$ where $\lambda_{0}$ usually corresponds to the maximum loading rate or margin of endurance in p.u, \%, MW or MVA depending on how the load variation is defined. Based on the bifurcation theory, two essential tools have been defined and applied to calculate this point of collapse: the direct and the continuation method. In the stress failure study, the continuation method shows many advantages because most researchers use it to track various bus power test systems' voltage profile to the change in the loading rate $\lambda$, namely Continuation Power Flow (CPF) [7]. In this research, the $\mathrm{CPF}$ algorithm with smooth changes in the load levels on various system buses was selected for simulation purposes. Two types of FACTS devices are considered in this study, SVC, and STATCOM. Details, including the basic structure and terminal characteristics of this FACTS device, are presented in the following sections.

\section{B. Shunt FACT Controller}

In this study one type of popular Shunt FACTS controller is implemented namely Static Var Compensator (SVC). The controller can maintain or control certain power system variables. This device is a static Var generator/load which is shunt connected, and its output is adjusted to exchange capacitive or inductive currents. The terminal bus voltage is usually the power system control variable. The popular SVC configuration consists of two configurations, namely a fixed capacitor (FC) and thyristor controlled reactor (TCR) configuration, as well as a thyristor switch capacitor (TSC) and TCR configuration [8]. The SVC behaves as a capacitor or inductor set within the minimum or maximum susceptance limits. One of the important problems in implementing SVC in increasing stress stability is selecting the appropriate size. The basic structure and characteristics of the SVC terminals used in this study are shown in Figs. 2 and 3 , respectively.

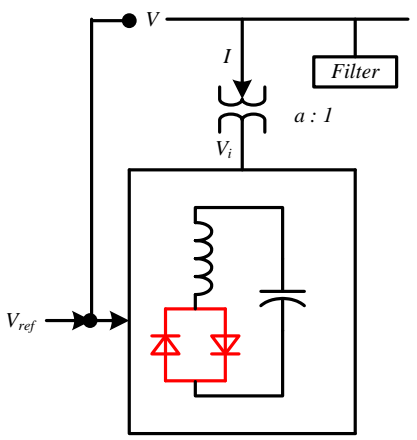

Fig. 2. The basic structure of SVC.

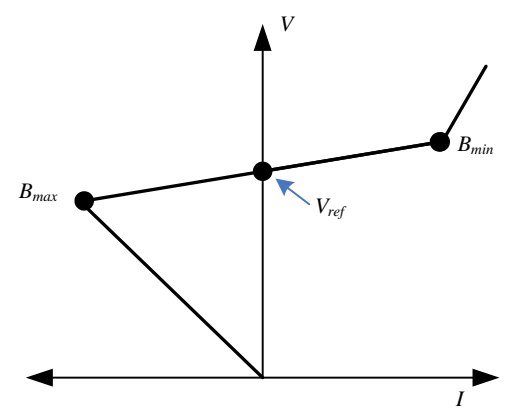

Fig. 3. Characteristics of SVC.

By performing Fourier series analysis on the inductor current waveform and assuming the control voltage is equal to the bus voltage, the TCR at the fundamental frequency can be considered to act like a variable inductance given by [9], [10]:

$$
X_{V}=X_{L} \frac{\pi}{2(\pi-\alpha)+\sin 2 \alpha}
$$

where $X_{L}$ and $\alpha$ are the reactants caused by the fundamental frequency without thyristor control and firing angle. Thus, the total controller equivalent impedance is defined as:

$$
X_{e}=X_{c} \frac{\pi / r_{x}}{\sin 2 \alpha-2 \alpha+\pi\left(2-\frac{1}{r_{s}}\right)}
$$

where $r_{x}=X_{C} / X_{L}$. The control limit is determined by the limit of the firing angle, which is set by design. Fig. 2 shows the typical steady-state control law of the SVC used in this study which the following current-voltage characteristics can represent:

$$
V=X_{\text {ref }}+X_{S L} I
$$

where $V$ and $I$ are the total RMS voltage and current magnitude, respectively, and $V_{\text {ref }}$ represents the reference voltage.

\section{RESEARCH METHODS}

\section{A. CPF Simulation}

Position The research method is to perform simulations using the Power System Analysis Toolbox (PSAT) simulation software [11], which has various features, including power flow and continues power flow (CPF). Using the CPF feature of the PSAT, the voltage stability of 
the test system can be investigated. The test system's behavior with and without the shunt FACTS device under different loading conditions was analyzed, which were expressed as Case-2 and Case-1, respectively. The location of the Shunt FACTS controllers was determined through bifurcation analysis. The typical PQ model is used on loads where the generator limit is negligible. The stress stability analysis is carried out by starting from the initial stable operating point and then increasing the load by a factor of $\lambda$ until a single point of electric flow is passed. The load is defined as:

$$
\begin{aligned}
& P_{L}=P_{L 0}(1+\lambda) \\
& Q_{L}=Q_{L 0}(1+\lambda)
\end{aligned}
$$

where $P_{L O}$ and $Q_{L O}$ are the active and reactive base loads, while $P_{L}$, and $Q_{L}$, are the active and reactive loads on bus $L$, respectively. The current operating point is defined by $\lambda$.

\section{1) Case-1 base case (without SVC)}

By using the CPF, critical buses are attained. The bus with the weakest [18] voltage profile will be obtained among the critical buses, represented by the PV curve for the tested system without the Shunt FACTS. This system presents the collapse or MLP, where the Jacobian matrix system becomes singular at $\lambda_{\max }$, expressed in p.u. The most extensive entries in the right and left eigenvectors associated with the zero eigenvalues at the bus's point of failure are indicated as a "critical voltage bus" requiring reactive power $Q$ support. The magnitude of the voltage across the MLP on the bus is known as the weakest bus.

\section{2) Case-2 with SVC}

In Case-2, one type of Shunt FACTS, namely SVC, is installed on the weakest bus, which is the lowest critical point, and repeat the simulation point 1) above. When the SVC is connected to the lowest bus, it will be possible to examine each bus's voltage profile in the test system and check the increase in loading until it reaches MLP at $\lambda_{\max }$. Then repeat the process by moving the same SVC to the next weakest bus and compare all the MLP results to get the most considerable $\lambda_{\max }$ value.

\section{B. CPF simulation}

In this study, the Bali 16-bus system was used as a practical test system to increase static voltage stability with the optimal placement of SVC, whose single line diagram is shown in Figs. 6 and 7. This test system consists of 4 bus generators located on buses $1,2,5$, and 6 , with a total generating capacity (capable of supplying) of 1282.6 MW, excluding the electricity supply from Banyuwangi through the $150 \mathrm{kV}$ Java-Bali interconnection line via submarine cable. The total active and reactive peak loads used in this simulation are 1239, $6 \mathrm{MW}$, and 286.4 MVAR, respectively. As discussed in the previous chapter, the type of FACTS used in this simulation is SVC, which can inject both reactive powers into the grid system.

\section{RESUlTS AND Discussion}

Modification of the Bali 16-bus practical test system is modeled using the PSAT power system analysis toolbox, as shown in the single line diagram of Fig. 4. The Shunt FACTS control device used, namely SVC in system testing, is also modeled using PSAT. The results were tested through two cases, namely (a) case-1, base case without SVC; then, the result is compared with (b) case-2, with SVC Case-1 base case (without shunt FACTS).

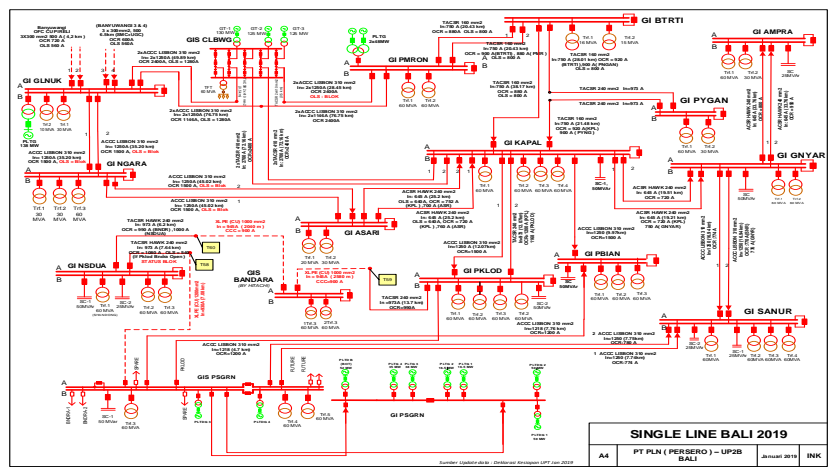

Fig. 4. Single line diagram of the Bali 16-bus system.

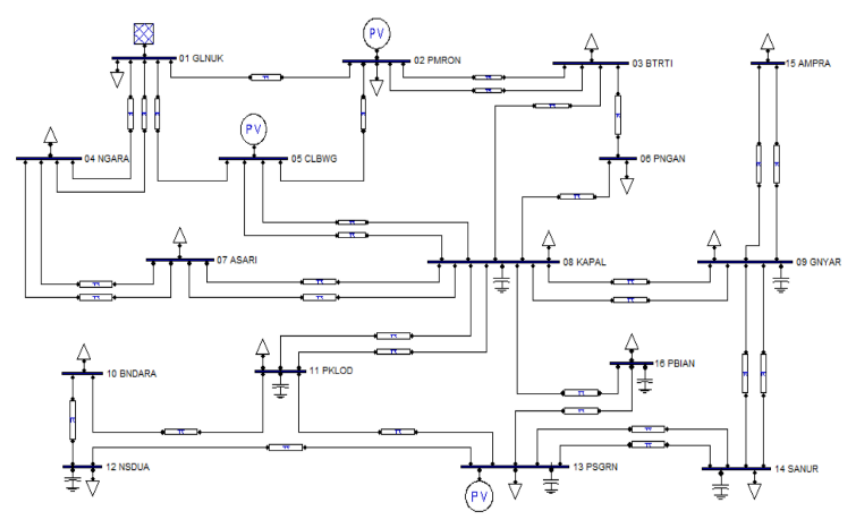

Fig. 5. Single line diagram of the modified Bali 16-bus system.

The results of the CPF power flow in the base case condition are shown in Fig. 6. Figure 6 shows that there are eight critical buses whose voltage values are less than 0.95 p.u. namely buses 04, 06, 07, 08, 09, 14, 15 and 16. Among these buses, bus-07 ASARI has the weakest / critical voltage profile of 0.678 p.u.

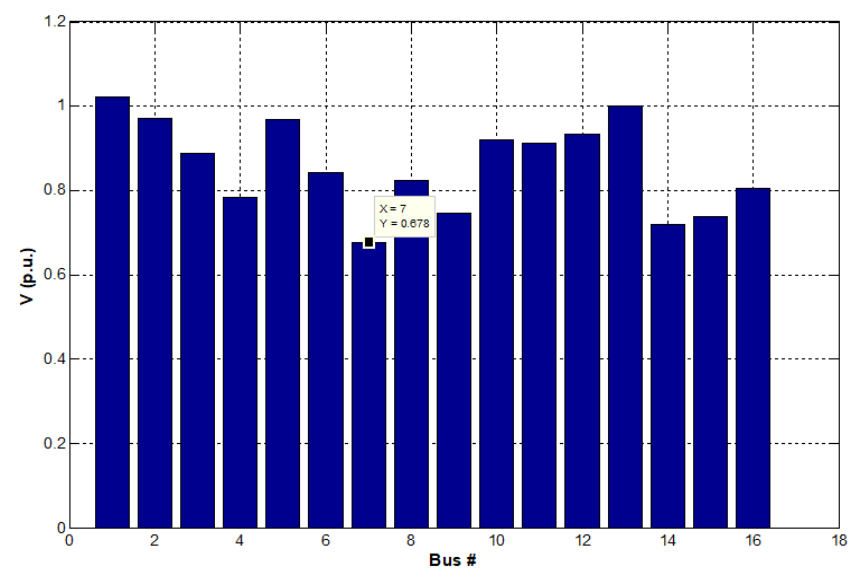

Fig. 6. Voltage profile for the Bali 16-bus test system without SVC.

Based on the analysis of Case-1 above, by installing an SVC on the most critical bus voltage, namely bus-07 ASARI, the voltage profile is obtained as depicted in Fig. 7. While Figs. 8 and 9 show the voltage profile, and the new MLP in Case2 is at $\lambda_{\max }=1,879$ p.u., which means an increase of 0.04 p.u. $(2.18 \%)$ compared to Case- 1 . 


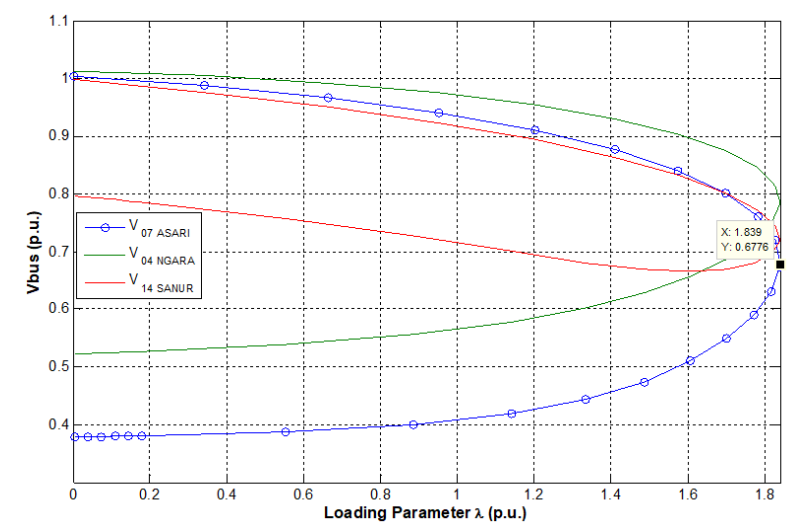

Figure 7. PV curve for the Bali 16-bus test system without SVC

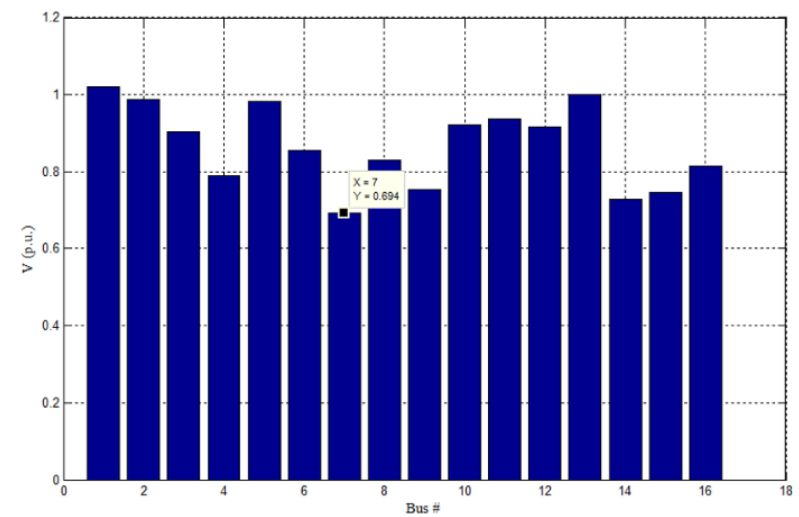

Fig. 8. Voltage profile for the 16-bus Bali test system with SVC on bus07.

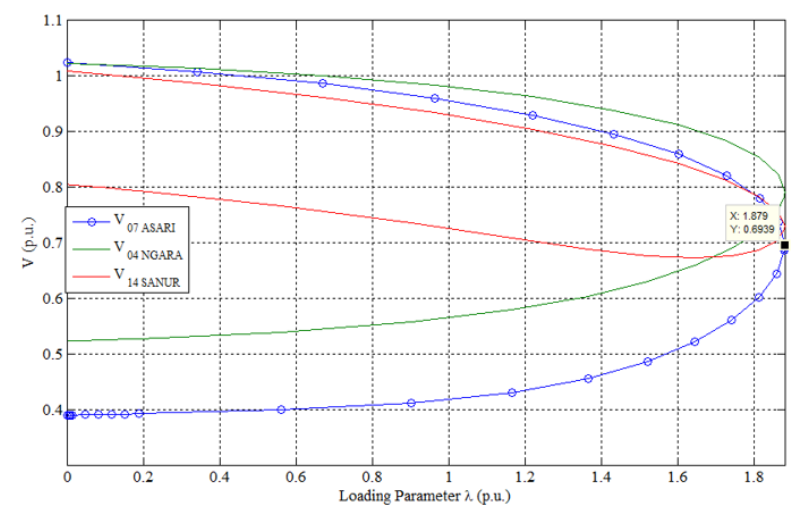

Fig. 9. PV curve for the 16-bus Bali Electrical test system with SVC on bus-07.

Figure 6 shows the three lowest bus voltage PV curves for the Bali 16-bus test system without SVC, i.e., bus-04 NGARA, bus-07 ASARI, and bus-14 SANUR. The system presents a collapse or MLP, where the Jacobian matrix system becomes single at $\lambda_{\text {maks }}=1,839$ p.u. Based on the largest entries in the right and left eigenvectors associated with the zero eigenvalues at the point of collapse, bus-07 ASARI is indicated as a critical voltage bus requiring an injection of reactive power $Q$. The MLP's voltage on bus-07 ASARI, known as the weakest bus, is $V_{\text {bus }}=0.6776$ p.u.

\section{A. Case-2 with SVC}

Figures 11 and 13 show that the MLP value does not increase more when the same SVC is transferred to another critical bus which is not the bus with the most critical voltage or the weakest bus, such as bus 04 bus 14 . The MLP result $\left(\lambda_{\max }\right)$ is lower than the placement on the bus-07, i.e., 1,854 p.u. and 1,867 p.u. as presented in Table 1. From Table 1 can also be observed that the lowest and the highest voltage profile can be achieved when placing the same SVC on bus04 and bus-07 as depicted on Figs. 10 and 12, respectively.

TABLE I. Simulation RESUlts For Both CASES

\begin{tabular}{cccc}
\hline Cases & Location & $\mathrm{X}[\lambda$ (p.u.)] & $\mathrm{Y}[\mathrm{V}(\mathrm{p} . u)]$. \\
\hline $\begin{array}{c}\text { Case-1 } \\
\text { base case }\end{array}$ & & 1.839 & 0.6776 \\
\hline \multirow{2}{\text{Case-2,with}}{\begin{tabular}{c} 
SVC \\
\cline { 2 - 4 }
\end{tabular}} & 7 & 1.879 & 0.6939 \\
\hline & 4 & 1.867 & 0.6652 \\
\hline
\end{tabular}

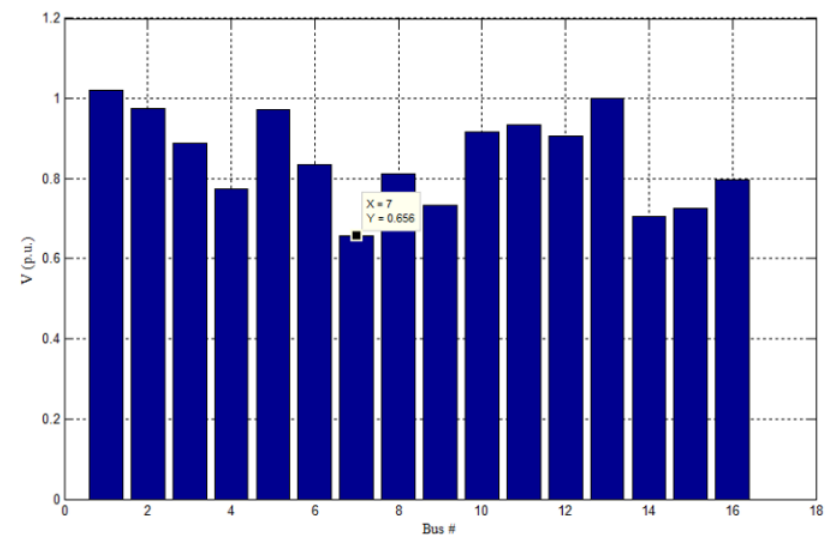

Fig. 10. Voltage profile for the 16-bus Bali electrical test system with SVC on bus 04 .

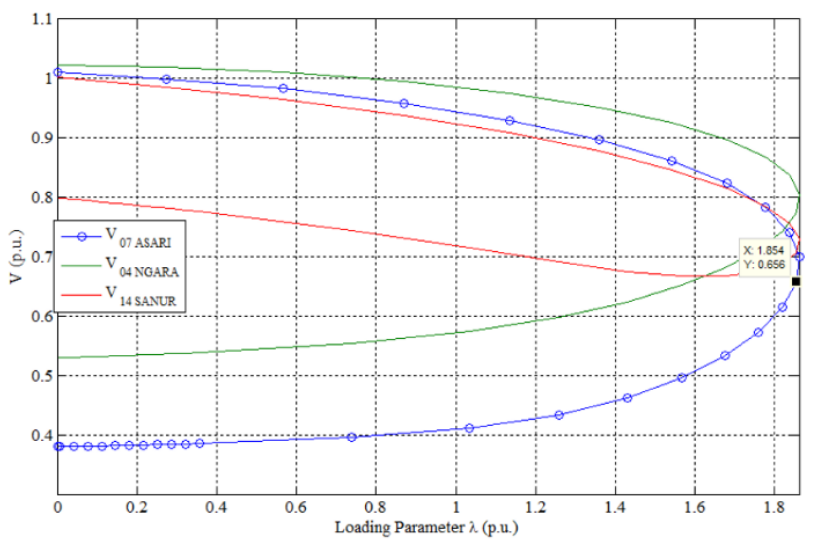

Fig. 11. PV curve for the Bali 16-bus system test with SVC on bus 04.

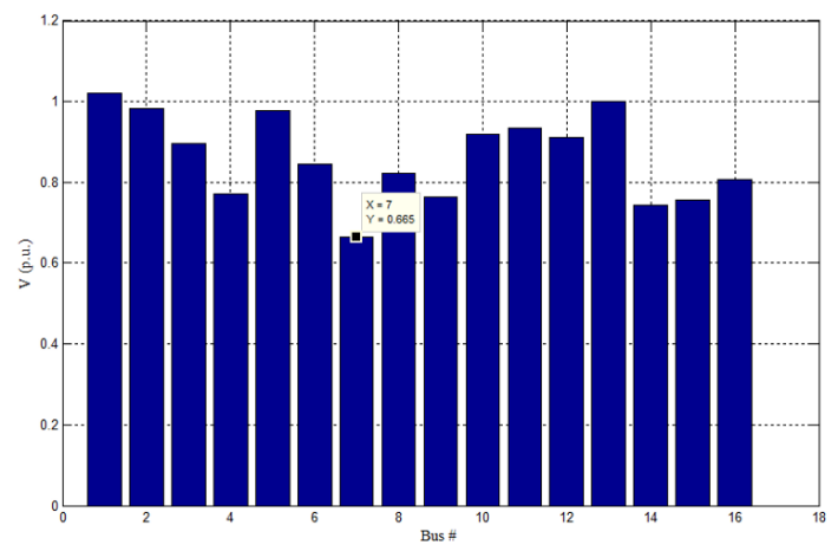

Fig. 12. Voltage profile for the 16-bus Bali test system with SVC on bus14. 


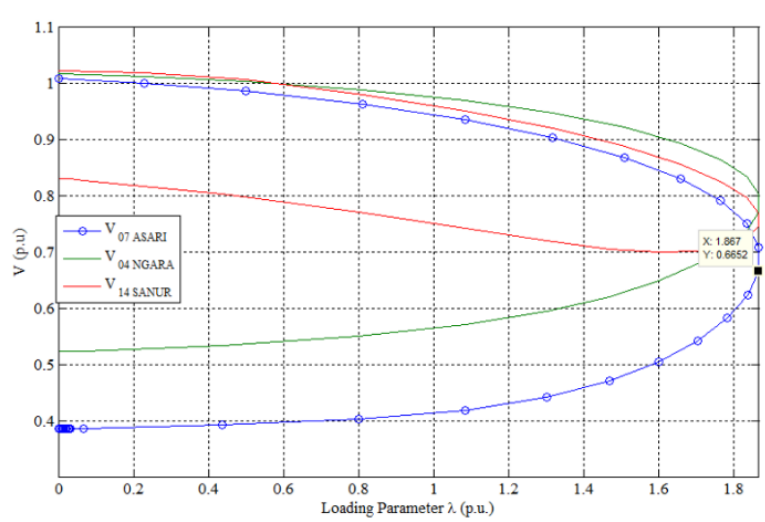

Fig. 13. PV curve for the Bali 16-bus system test with SVC on bus-14.

\section{CONCLUSION}

This study thrived in determining the best location for installing one type of Shunt FACTS, namely SVC on the Bali 16-bus test system at the weakest voltage location, i.e., bus-07 ASARI, to obtain the maximum level or largest voltage statistic of $\lambda_{\max }=1.879$ p.u. Installing the SVC in the best location can increase MLP by $2.18 \%$ compared to case1 , base case. However, the lowest voltage profile on bus-07 by installing the SVC resulted in a higher voltage drop of 0.694 p.u compared to case-1, base case. The results presented in this study clearly show that installing a Shunt FACTS in the best location can improve the loading capability of the Bali 16-bus system, which is expressed with a maximum load point of close to $2 \%$ compared to the base case or without the FACTS installation.

\section{ACKNOWLEDGMENT}

The author expresses his gratitude to National Institute of Technology, Malang for assisting this research through the Institute for Research and Community Service fiscal year 2019.

\section{REFERENCES}

[1] C. J. Parker, I. F. Morrison, and D. Sutanto, "Application of an optimisation method for determining the reactive margin from voltage collapse in reactive power planning," IEEE Transactions on Power Systems, vol. 11, no. 3, pp. 1473-1481, 1996, doi: $10.1109 / 59.535688$.

[2] R. Gan, Z. Luan, Y. Yang, W. Liu, and S. Yang, "Static voltage stability analysis based on improved continuous power flow," in TENCON 2015-2015 IEEE Region 10 Conference, 2015: IEEE, pp. 1-3.

[3] N. Voropai, N. Tomin, V. Kurbatsky, D. Panasetsky, D. Sidorov, and A. Zhukov, "Development of computional intelligence-based algorithms of preventing voltage collapse in power systems with a complex multi-loop structure," in 2016 IEEE PES Asia-Pacific Power and Energy Engineering Conference (APPEEC), 25-28 Oct. 2016 2016, pp. 1-5, doi: 10.1109/APPEEC.2016.7827553.

[4] I. Dobson and L. Lu, "Voltage collapse precipitated by the immediate change in stability when generator reactive power limits are encountered," IEEE Transactions on Circuits and Systems I: Fundamental Theory and Applications, vol. 39, no. 9, pp. 762-766, 1992, doi: 10.1109/81.250167.
[5] N. Talebi, M. Ehsan, and S. Bathaee, "Effects of SVC and TCSC control strategies on static voltage collapse phenomena," in SoutheastCon, 2004. Proceedings. IEEE, 2004: IEEE, pp. 161-168.

[6] A. Singhal and V. Ajjarapu, "Long-term voltage stability assessment of an integrated transmission distribution system," in 2017 North American Power Symposium (NAPS), 2017: IEEE, pp. 1-6.

[7] B. S. Adusumilli and B. K. Kumar, "Modified affine arithmetic based continuation power flow analysis for voltage stability assessment under uncertainty," IET Generation, Transmission \& Distribution, vol. 12, no. 18, pp. 4225-4232, 2018.

[8] A. Pathak, M. Sharma, and M. Gupta, "Modeling and simulation of SVC for reactive power control in high penetration wind power system," in 2015 Annual IEEE India Conference (INDICON), 2015: IEEE, pp. 1-6.

[9] M. A. Kamarposhti and M. Alinezhad, "Comparison of SVC and STATCOM in static voltage stability margin enhancement," system, vol. 9 , p. 1,2010

[10] S. S. Bhole and P. Nigam, "Improvement of voltage stability in power system by using SVC and STATCOM," International Journal of Advanced Research in Electrical, Electronics and Instrumentation Engineering, vol. 4, no. 2, pp. 76-81, 2015.

[11] F. Milano, L. Vanfretti, and J. C. Morataya, "An Open Source Power System Virtual Laboratory: The PSAT Case and Experience," IEEE Transactions on Education, vol. 51, no. 1, pp. 17-23, 2008, doi: 10.1109/TE.2007.893354.

\section{BIOGRAPHIES}

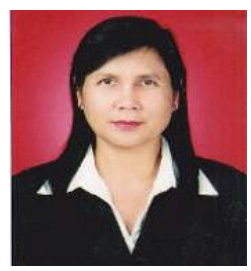

Ni Putu Agustini obtained her B. Eng. from National Institute of Technology, Malang and her M. Eng. Brawijaya University, Malang both in electrical engineering in 1986 and 2008, respectively. Currently, she is working as a lecturer for undergraduate students in the Department of Electrical Engineering, National Institute of Technology, Malang. Her research interests include integrating renewable energy into the power grid and the application of reliability to power systems.

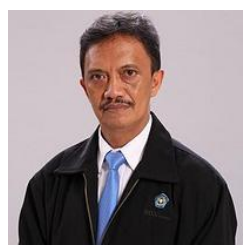

I Made Wartana obtained his B. Eng from National Institute of Technology, Malang in 1987 and his M Eng. From Bandung Institute of Technology, Bandung in 1995 both in electrical engineering. His Doctor of Engineering earned from Asian Institute of Technology Thailand with specialization in Electric Power Systems Management in 2012. Currently, Dr. Made is a full professor in the Department of Electrical Engineering, National Institute of Technology, Malang. His research interests include the application of FACTS controllers to the power grid, the application of AI to power systems, and the integration of renewable energies with the grid.

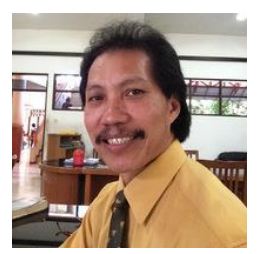

Abraham Lomi obtained his B. Eng. from National Institute of Technology, Malang and his M. Eng. From Bandung Institute of Technology Bandung both in electrical engineering in 1987 and 1992 respectively. The degree of Doctor of Engineering earned from Asian Institute of Technology, Thailand in 2000. Currently, Dr. Lomi is a full professor in the Department of Electrical Engineering and Director of Renewable Energy Research Center. His research interests include power system dynamic and stability, power electronics, power quality, renewable energy, and smart grid. 